\title{
Supplemental information for: Determining fingerprint age with mass spectrometry imaging via ozonolysis of triacylglycerols
}

\author{
Paige Hinners, Madison Thomas, Young-Jin Lee* \\ Department of Chemistry, lowa State University, Ames, lowa 50011 \\ *Correspondence to:
}

Tel: 515-294-1235, Email: yjlee@iastate.edu

This document contains both supplemental tables and figures. The supplemental tables (S-1 through S-3) include the compounds tracked and utilized in this study. Figure S-1, S-3, and S-4 contain mass spectra that support information presented in the main text. Figure $S-2$ is a representative graph of the temperature and humidity while the studies were performed. 
Table S-1. List of all triacylglycerols (TGs) detected and used in this study along with their theoretical and experimental $\mathrm{m} / \mathrm{z}$ values and mass errors. Red and blue indicate saturated and unsaturated TGs, respectively.

\begin{tabular}{|c|c|c|c|c|c|c|c|c|c|}
\hline \multicolumn{10}{|c|}{ Triacylglycerols } \\
\hline TG & Formula & $\begin{array}{l}\text { Theoretical } \\
\qquad(\mathrm{M}+\mathrm{Na})\end{array}$ & $\begin{array}{l}\text { Experimental } \\
\qquad(\mathrm{M}+\mathrm{Na})\end{array}$ & $\underset{(p p m)}{\Delta m}$ & TG & Formula & $\begin{array}{l}\text { Theoretical } \\
\qquad(\mathrm{M}+\mathrm{Na})\end{array}$ & $\begin{array}{l}\text { Experimental } \\
\qquad(\mathrm{M}+\mathrm{Na})\end{array}$ & $\underset{(p p m)}{\Delta m}$ \\
\hline TG 42:3 & $\mathrm{C}_{45} \mathrm{H}_{80} \mathrm{O}_{6}$ & 739.585 & 739.585 & -0.1 & TG 48:4 & $\mathrm{C}_{51} \mathrm{H}_{90} \mathrm{O}_{6}$ & 821.663 & 821.663 & -0.4 \\
\hline TG 42:2 & $\mathrm{C}_{45} \mathrm{H}_{82} \mathrm{O}_{6}$ & 741.600 & 741.600 & 0.1 & TG 48:3 & $\mathrm{C}_{51} \mathrm{H}_{92} \mathrm{O}_{6}$ & 823.679 & 823.679 & -0.2 \\
\hline TG 42:1 & $\mathrm{C}_{45} \mathrm{H}_{84} \mathrm{O}_{6}$ & 743.616 & 743.616 & -0.1 & TG 48:2 & $\mathrm{C}_{51} \mathrm{H}_{94} \mathrm{O}_{6}$ & 825.694 & 825.694 & -0.2 \\
\hline TG 42:0 & $\mathrm{C}_{45} \mathrm{H}_{86} \mathrm{O}_{6}$ & 745.632 & 745.632 & -0.2 & TG 48:1 & $\mathrm{C}_{51} \mathrm{H}_{96} \mathrm{O}_{6}$ & 827.710 & 827.710 & -0.2 \\
\hline TG 43:4 & $\mathrm{C}_{46} \mathrm{H}_{80} \mathrm{O}_{6}$ & 751.585 & 751.584 & 0.8 & TG 48:0 & $\mathrm{C}_{51} \mathrm{H}_{98} \mathrm{O}_{6}$ & 829.726 & 829.725 & 0.6 \\
\hline TG 43:3 & $\mathrm{C}_{46} \mathrm{H}_{82} \mathrm{O}_{6}$ & 753.600 & 753.601 & -0.2 & TG 49:4 & $\mathrm{C}_{52} \mathrm{H}_{92} \mathrm{O}_{6}$ & 835.679 & 835.679 & -0.3 \\
\hline TG 43:2 & $\mathrm{C}_{46} \mathrm{H}_{84} \mathrm{O}_{6}$ & 755.616 & 755.616 & -0.2 & TG 49:3 & $\mathrm{C}_{52} \mathrm{H}_{94} \mathrm{O}_{6}$ & 837.694 & 837.695 & -0.3 \\
\hline TG 43:1 & $\mathrm{C}_{46} \mathrm{H}_{86} \mathrm{O}_{6}$ & 757.632 & 757.632 & -0.6 & TG 49:2 & $\mathrm{C}_{52} \mathrm{H}_{96} \mathrm{O}_{6}$ & 839.710 & 839.710 & -0.2 \\
\hline TG 43:0 & $\mathrm{C}_{46} \mathrm{H}_{88} \mathrm{O}_{6}$ & 759.647 & 759.648 & -0.2 & TG 49:1 & $\mathrm{C}_{52} \mathrm{H}_{98} \mathrm{O}_{6}$ & 841.726 & 841.726 & 0.1 \\
\hline TG 44:3 & $\mathrm{C}_{47} \mathrm{H}_{84} \mathrm{O}_{6}$ & 767.616 & 767.616 & -0.1 & TG 49:0 & $\mathrm{C}_{52} \mathrm{H}_{100} \mathrm{O}_{6}$ & 843.741 & 843.740 & 1.7 \\
\hline TG 44:2 & $\mathrm{C}_{47} \mathrm{H}_{86} \mathrm{O}_{6}$ & 769.632 & 769.632 & -0.3 & TG 50:4 & $\mathrm{C}_{53} \mathrm{H}_{94} \mathrm{O}_{6}$ & 849.694 & 849.694 & -0.2 \\
\hline TG 44:1 & $\mathrm{C}_{47} \mathrm{H}_{88} \mathrm{O}_{6}$ & 771.647 & 771.648 & -0.4 & TG 50:3 & $\mathrm{C}_{53} \mathrm{H}_{96} \mathrm{O}_{6}$ & 851.710 & 851.710 & -0.2 \\
\hline TG 44:0 & $\mathrm{C}_{47} \mathrm{H}_{90} \mathrm{O}_{6}$ & 773.663 & 773.663 & -0.2 & TG 50:2 & $\mathrm{C}_{53} \mathrm{H}_{98} \mathrm{O}_{6}$ & 853.726 & 853.726 & 0.0 \\
\hline TG 45:4 & $\mathrm{C}_{48} \mathrm{H}_{84} \mathrm{O}_{6}$ & 779.616 & 779.616 & -0.2 & TG 50:1 & $\mathrm{C}_{53} \mathrm{H}_{100 \mathrm{O}_{6}}$ & 855.741 & 855.741 & 0.5 \\
\hline TG 45:3 & $\mathrm{C}_{48} \mathrm{H}_{86} \mathrm{O}_{6}$ & 781.632 & 781.632 & -0.2 & TG 50:0 & $\mathrm{C}_{53} \mathrm{H}_{102} \mathrm{O}_{6}$ & 857.757 & 857.755 & 2.6 \\
\hline TG 45:2 & $\mathrm{C}_{48} \mathrm{H}_{88} \mathrm{O}_{6}$ & 783.647 & 783.648 & -0.2 & TG 51:4 & $\mathrm{C}_{54} \mathrm{H}_{96} \mathrm{O}_{6}$ & 863.710 & 863.710 & 0.1 \\
\hline TG 45:1 & $\mathrm{C}_{48} \mathrm{H}_{90} \mathrm{O}_{6}$ & 785.663 & 785.663 & -0.4 & TG 51:3 & $\mathrm{C}_{54} \mathrm{H}_{98} \mathrm{O}_{6}$ & 865.726 & 865.726 & 0.1 \\
\hline TG 45:0 & $\mathrm{C}_{48} \mathrm{H}_{92} \mathrm{O}_{6}$ & 787.679 & 787.679 & -0.4 & TG 51:2 & $\mathrm{C}_{54} \mathrm{H}_{100} \mathrm{O}_{6}$ & 867.741 & 867.741 & 0.2 \\
\hline TG 46:4 & $\mathrm{C}_{49} \mathrm{H}_{86} \mathrm{O}_{6}$ & 793.632 & 793.632 & -0.5 & TG 51:1 & $\mathrm{C}_{54} \mathrm{H}_{102} \mathrm{O}_{6}$ & 869.757 & 869.756 & 1.1 \\
\hline TG 46:3 & $\mathrm{C}_{49} \mathrm{H}_{88} \mathrm{O}_{6}$ & 795.647 & 795.648 & -0.4 & TG 51:0 & $\mathrm{C}_{54} \mathrm{H}_{104} \mathrm{O}_{6}$ & 871.773 & 871.769 & 3.9 \\
\hline TG 46:2 & $\mathrm{C}_{49} \mathrm{H}_{90} \mathrm{O}_{6}$ & 797.663 & 797.663 & -0.2 & TG 52:4 & $\mathrm{C}_{55} \mathrm{H}_{9806}$ & 877.726 & 877.726 & -0.2 \\
\hline TG 46:1 & $\mathrm{C}_{49} \mathrm{H}_{92} \mathrm{O}_{6}$ & 799.679 & 799.679 & -0.4 & TG 52:3 & $\mathrm{C}_{55} \mathrm{H}_{100} \mathrm{O}_{6}$ & 879.741 & 879.741 & -0.2 \\
\hline TG 46:0 & $\mathrm{C}_{49} \mathrm{H}_{94} \mathrm{O}_{6}$ & 801.694 & 801.694 & 0.1 & TG 52:2 & $\mathrm{C}_{55} \mathrm{H}_{102} \mathrm{O}_{6}$ & 881.757 & 881.757 & 0.3 \\
\hline TG 47:4 & $\mathrm{C}_{50} \mathrm{H}_{88} \mathrm{O}_{6}$ & 807.647 & 807.648 & -0.4 & TG 52:1 & $\mathrm{C}_{55} \mathrm{H}_{104} \mathrm{O}_{6}$ & 883.773 & 883.772 & 1.1 \\
\hline TG 47:3 & $\mathrm{C}_{50} \mathrm{H}_{90} \mathrm{O}_{6}$ & 809.663 & 809.663 & -0.4 & TG 52:0 & $\mathrm{C}_{55} \mathrm{H}_{106} \mathrm{O}_{6}$ & 885.788 & 885.787 & 1.7 \\
\hline TG 47:2 & $\mathrm{C}_{50} \mathrm{H}_{92} \mathrm{O}_{6}$ & 811.679 & 811.679 & -0.2 & & & & & \\
\hline TG 47:1 & $\mathrm{C}_{50} \mathrm{H}_{94} \mathrm{O}_{6}$ & 813.694 & 813.694 & -0.2 & & & & & \\
\hline TG 47:0 & $\mathrm{C}_{50} \mathrm{H}_{96} \mathrm{O}_{6}$ & 815.710 & 815.710 & 0.3 & & & & & \\
\hline
\end{tabular}


Table S-2. List of all 07 series compounds detected and used in this study along with their theoretical and experimental $\mathrm{m} / \mathrm{z}$ values and mass errors.

\begin{tabular}{|l|c|c|c|}
\hline \multicolumn{4}{|c|}{ O7 Series } \\
\hline Formula & $\begin{array}{c}\text { Theoretical } \\
\text { (M+Na) }\end{array}$ & $\begin{array}{c}\text { Experimental } \\
\text { (M+Na) }\end{array}$ & $\begin{array}{c}\Delta \mathbf{m} \\
\text { (ppm) }\end{array}$ \\
\hline $\mathrm{C}_{36} \mathrm{H}_{64} \mathrm{O}_{7}$ & 631.454 & 631.452 & 4.3 \\
\hline $\mathrm{C}_{36} \mathrm{H}_{66} \mathrm{O}_{7}$ & 633.470 & 633.468 & 3.8 \\
\hline $\mathrm{C}_{37} \mathrm{H}_{66} \mathrm{O}_{7}$ & 645.470 & 645.468 & 3.5 \\
\hline $\mathrm{C}_{37} \mathrm{H}_{68} \mathrm{O}_{7}$ & 647.486 & 647.483 & 3.8 \\
\hline $\mathrm{C}_{38} \mathrm{H}_{68} \mathrm{O}_{7}$ & 659.486 & 659.484 & 2.9 \\
\hline $\mathrm{C}_{38} \mathrm{H}_{70} \mathrm{O}_{7}$ & 661.501 & 661.499 & 3.4 \\
\hline $\mathrm{C}_{39} \mathrm{H}_{70} \mathrm{O}_{7}$ & 673.501 & 673.499 & 3.8 \\
\hline $\mathrm{C}_{39} \mathrm{H}_{72} \mathrm{O}_{7}$ & 675.517 & 675.515 & 3.4 \\
\hline $\mathrm{C}_{40} \mathrm{H}_{72} \mathrm{O}_{7}$ & 687.517 & 687.515 & 3.2 \\
\hline $\mathrm{C}_{40} \mathrm{H}_{74} \mathrm{O}_{7}$ & 689.533 & 689.530 & 3.5 \\
\hline $\mathrm{C}_{41} \mathrm{H}_{74} \mathrm{O}_{7}$ & 701.533 & 701.530 & 3.4 \\
\hline $\mathrm{C}_{41} \mathrm{H}_{76} \mathrm{O}_{7}$ & 703.548 & 703.546 & 3.2 \\
\hline $\mathrm{C}_{42} \mathrm{H}_{76} \mathrm{O}_{7}$ & 715.548 & 715.546 & 3.3 \\
\hline $\mathrm{C}_{42} \mathrm{H}_{78} \mathrm{O}_{7}$ & 717.564 & 717.562 & 3.3 \\
\hline $\mathrm{C}_{43} \mathrm{H}_{78} \mathrm{O}_{7}$ & 729.564 & 729.562 & 3.0 \\
\hline $\mathrm{C}_{43} \mathrm{H}_{80} \mathrm{O}_{7}$ & 731.580 & 731.577 & 3.0 \\
\hline $\mathrm{C}_{44} \mathrm{H}_{80} \mathrm{O}_{7}$ & 743.580 & 743.578 & 2.9 \\
\hline $\mathrm{C}_{44} \mathrm{H}_{82} \mathrm{O}_{7}$ & 745.595 & 745.594 & 2.4 \\
\hline $\mathrm{C}_{45} \mathrm{H}_{82} \mathrm{O}_{7}$ & 757.595 & 757.594 & 2.3 \\
\hline $\mathrm{C}_{45} \mathrm{H}_{84} \mathrm{O}_{7}$ & 759.611 & 759.610 & 1.8 \\
\hline $\mathrm{C}_{46} \mathrm{H}_{84} \mathrm{O}_{7}$ & 771.611 & 771.609 & 2.4 \\
\hline $\mathrm{C}_{46} \mathrm{H}_{86} \mathrm{O}_{7}$ & 773.627 & 773.625 & 1.5 \\
\hline & & & \\
\hline
\end{tabular}


Table S-3. List of all 08 series compounds detected and used in this study along with their theoretical and experimental $\mathrm{m} / \mathrm{z}$ values and mass errors.

\begin{tabular}{|c|c|c|c|c|c|c|c|}
\hline \multicolumn{8}{|c|}{08 Series } \\
\hline Formula & $\begin{array}{l}\text { Theoretical } \\
(\mathrm{M}-\mathrm{H}+2 \mathrm{Na})\end{array}$ & $\begin{array}{c}\text { Experimental } \\
(\mathrm{M}-\mathrm{H}+2 \mathrm{Na})\end{array}$ & $\begin{array}{c}\Delta m \\
(p p m)\end{array}$ & Formula & $\begin{array}{c}\text { Theoretical } \\
(\mathrm{M}+\mathrm{Na})\end{array}$ & $\begin{array}{c}\text { Experimental } \\
(\mathrm{M}+\mathrm{Na})\end{array}$ & $\begin{array}{c}\Delta \mathrm{m} \\
(\mathrm{ppm})\end{array}$ \\
\hline $\mathrm{C}_{36} \mathrm{H}_{66} \mathrm{O}_{8}$ & 671.447 & 671.445 & 3.6 & $\mathrm{C}_{36} \mathrm{H}_{66} \mathrm{O}_{8}$ & 649.465 & 649.463 & 3.1 \\
\hline $\mathrm{C}_{37} \mathrm{H}_{66} \mathrm{O}_{8}$ & 683.447 & 683.445 & 3.1 & $\mathrm{C}_{37} \mathrm{H}_{66} \mathrm{O}_{8}$ & 661.465 & 661.463 & 3.3 \\
\hline $\mathrm{C}_{37} \mathrm{H}_{68} \mathrm{O}_{8}$ & 685.463 & 685.460 & 3.3 & $\mathrm{C}_{37} \mathrm{H}_{68} \mathrm{O}_{8}$ & 663.481 & 663.479 & 3.2 \\
\hline $\mathrm{C}_{38} \mathrm{H}_{68} \mathrm{O}_{8}$ & 697.463 & 697.461 & 2.9 & $\mathrm{C}_{38} \mathrm{H}_{68} \mathrm{O}_{8}$ & 675.481 & 675.478 & 3.3 \\
\hline $\mathrm{C}_{38} \mathrm{H}_{70} \mathrm{O}_{8}$ & 699.478 & 699.476 & 3.2 & $\mathrm{C}_{38} \mathrm{H}_{70} \mathrm{O}_{8}$ & 677.496 & 677.496 & 1.2 \\
\hline $\mathrm{C}_{39} \mathrm{H}_{70} \mathrm{O}_{8}$ & 711.478 & 711.476 & 2.7 & $\mathrm{C}_{39} \mathrm{H}_{70} \mathrm{O}_{8}$ & 689.496 & 689.495 & 2.6 \\
\hline $\mathrm{C}_{39} \mathrm{H}_{72} \mathrm{O}_{8}$ & 713.494 & 713.492 & 3.3 & $\mathrm{C}_{39} \mathrm{H}_{72} \mathrm{O}_{8}$ & 691.512 & 691.510 & 2.4 \\
\hline $\mathrm{C}_{40} \mathrm{H}_{72} \mathrm{O}_{8}$ & 725.494 & 725.492 & 2.9 & $\mathrm{C}_{40} \mathrm{H}_{72} \mathrm{O}_{8}$ & 703.512 & 703.510 & 2.2 \\
\hline $\mathrm{C}_{40} \mathrm{H}_{74} \mathrm{O}_{8}$ & 727.510 & 727.507 & 3.2 & $\mathrm{C}_{40} \mathrm{H}_{74} \mathrm{O}_{8}$ & 705.528 & 705.526 & 2.1 \\
\hline $\mathrm{C}_{41} \mathrm{H}_{74} \mathrm{O}_{8}$ & 739.510 & 739.507 & 3.2 & $\mathrm{C}_{41} \mathrm{H}_{74} \mathrm{O}_{8}$ & 717.528 & 717.527 & 1.2 \\
\hline $\mathrm{C}_{41} \mathrm{H}_{76} \mathrm{O}_{8}$ & 741.525 & 741.523 & 3.1 & $\mathrm{C}_{41} \mathrm{H}_{76} \mathrm{O}_{8}$ & 719.543 & 719.542 & 2.3 \\
\hline $\mathrm{C}_{42} \mathrm{H}_{76} \mathrm{O}_{8}$ & 753.525 & 753.523 & 3.4 & $\mathrm{C}_{42} \mathrm{H}_{76} \mathrm{O}_{8}$ & 731.543 & 731.541 & 2.5 \\
\hline $\mathrm{C}_{42} \mathrm{H}_{78} \mathrm{O}_{8}$ & 755.541 & 755.539 & 3.1 & $\mathrm{C}_{42} \mathrm{H}_{78} \mathrm{O}_{8}$ & 733.559 & 733.558 & 1.8 \\
\hline $\mathrm{C}_{43} \mathrm{H}_{78} \mathrm{O}_{8}$ & 767.541 & 767.539 & 2.5 & $\mathrm{C}_{43} \mathrm{H}_{78} \mathrm{O}_{8}$ & 745.559 & 745.557 & 2.1 \\
\hline $\mathrm{C}_{43} \mathrm{H}_{80} \mathrm{O}_{8}$ & 769.556 & 769.554 & 2.7 & $\mathrm{C}_{43} \mathrm{H}_{80} \mathrm{O}_{8}$ & 747.575 & 747.573 & 2.1 \\
\hline $\mathrm{C}_{44} \mathrm{H}_{80} \mathrm{O}_{8}$ & 781.556 & 781.555 & 2.0 & $\mathrm{C}_{44} \mathrm{H}_{80} \mathrm{O}_{8}$ & 759.575 & 759.572 & 3.7 \\
\hline $\mathrm{C}_{44} \mathrm{H}_{82} \mathrm{O}_{8}$ & 783.572 & 783.570 & 2.9 & $\mathrm{C}_{44} \mathrm{H}_{82} \mathrm{O}_{8}$ & 761.590 & 761.588 & 2.4 \\
\hline $\mathrm{C}_{45} \mathrm{H}_{82} \mathrm{O}_{8}$ & 795.572 & 795.569 & 4.2 & $\mathrm{C}_{45} \mathrm{H}_{82} \mathrm{O}_{8}$ & 773.590 & 773.589 & 1.5 \\
\hline $\mathrm{C}_{45} \mathrm{H}_{84} \mathrm{O}_{8}$ & 797.588 & 797.586 & 2.9 & $\mathrm{C}_{45} \mathrm{H}_{84} \mathrm{O}_{8}$ & 775.606 & 775.604 & 2.2 \\
\hline $\mathrm{C}_{46} \mathrm{H}_{86} \mathrm{O}_{8}$ & 811.603 & 811.601 & 3.1 & $\mathrm{C}_{46} \mathrm{H}_{86} \mathrm{O}_{8}$ & 789.621 & 789.622 & -0.5 \\
\hline
\end{tabular}




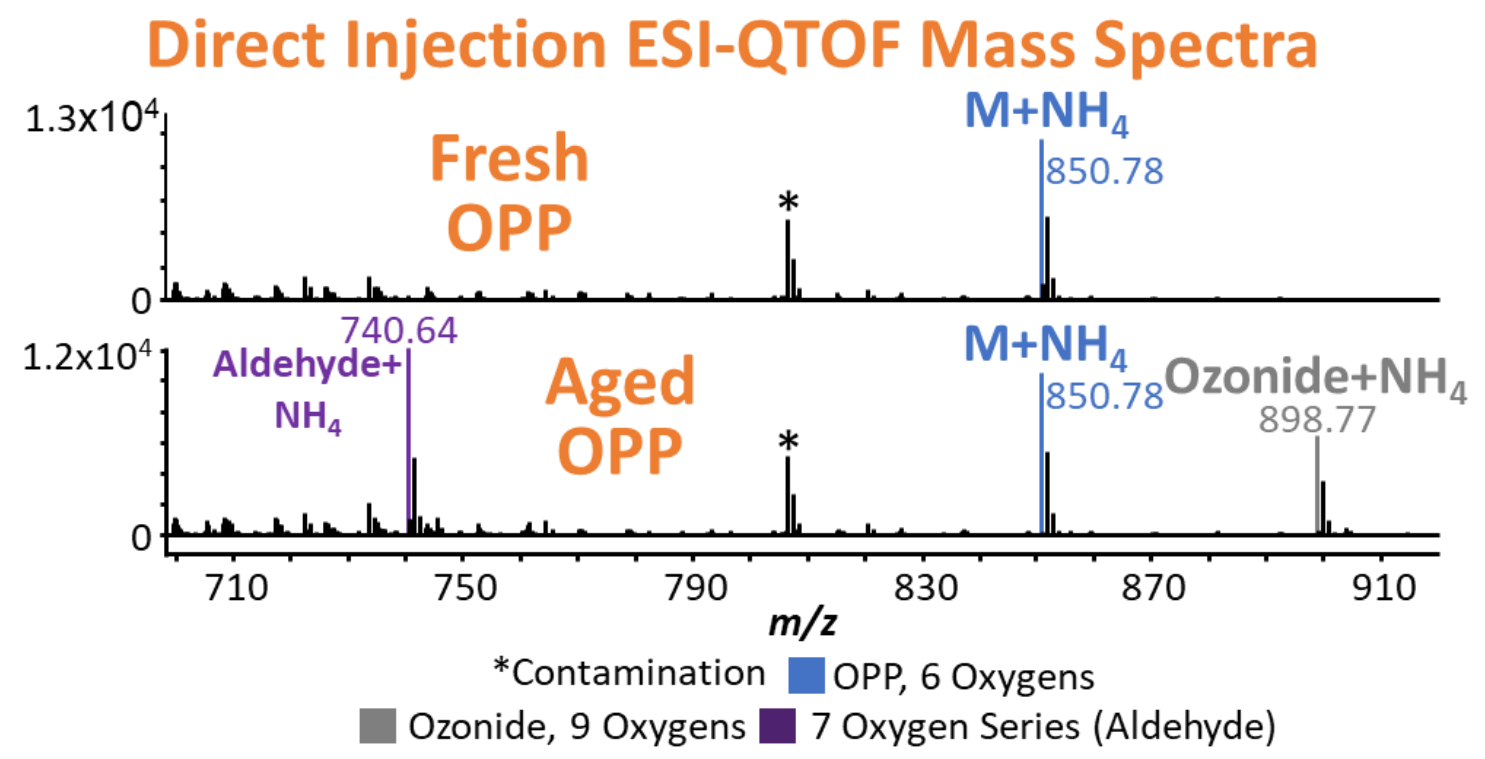

Figure S-1. ESI mass spectra of fresh and aged 1-oleoyl-2,3-dipalmitoyl glycerol (OPP) obtained using direct injection ESI QTOF.

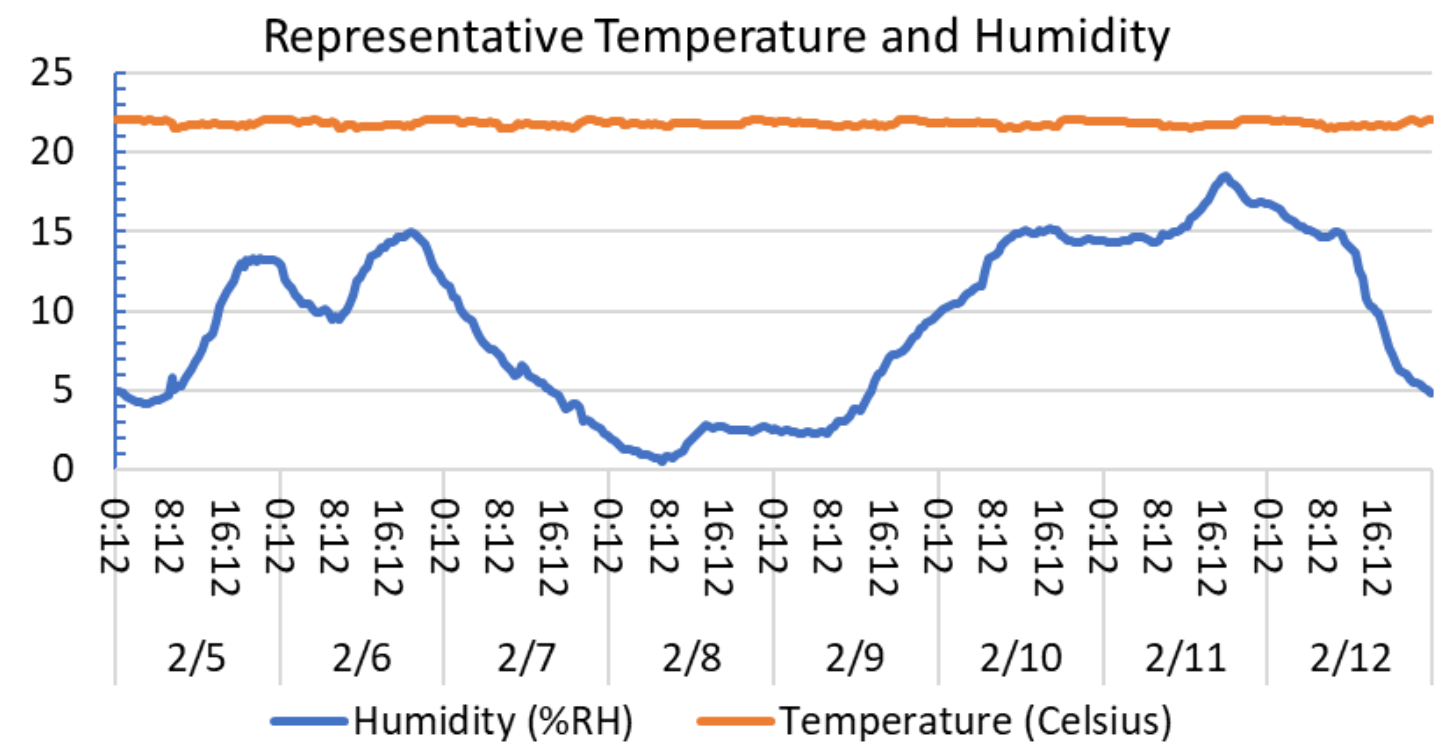

Figure S-2. Representative graph tracking percent relative humidity and temperature over the course of the aging study. 


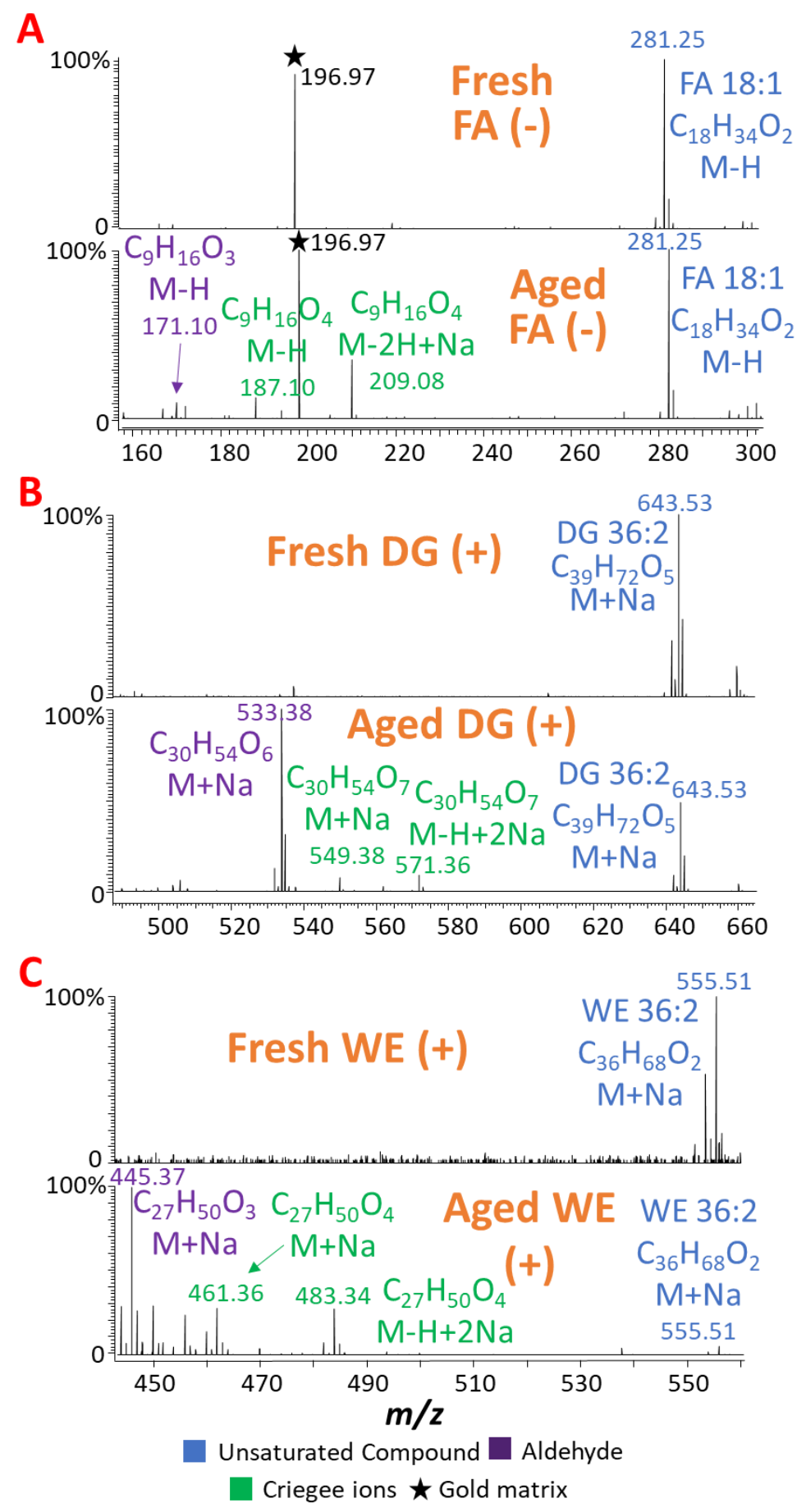

Figure S-3. Fresh and aged MALDI mass spectra of (A) oleic acid, a fatty acid, in negative mode (B) 1,2-dioleoyl-glycerol, a diacylglycerol, in positive mode, and (C) oleyl oleate, a wax ester in positive mode. 


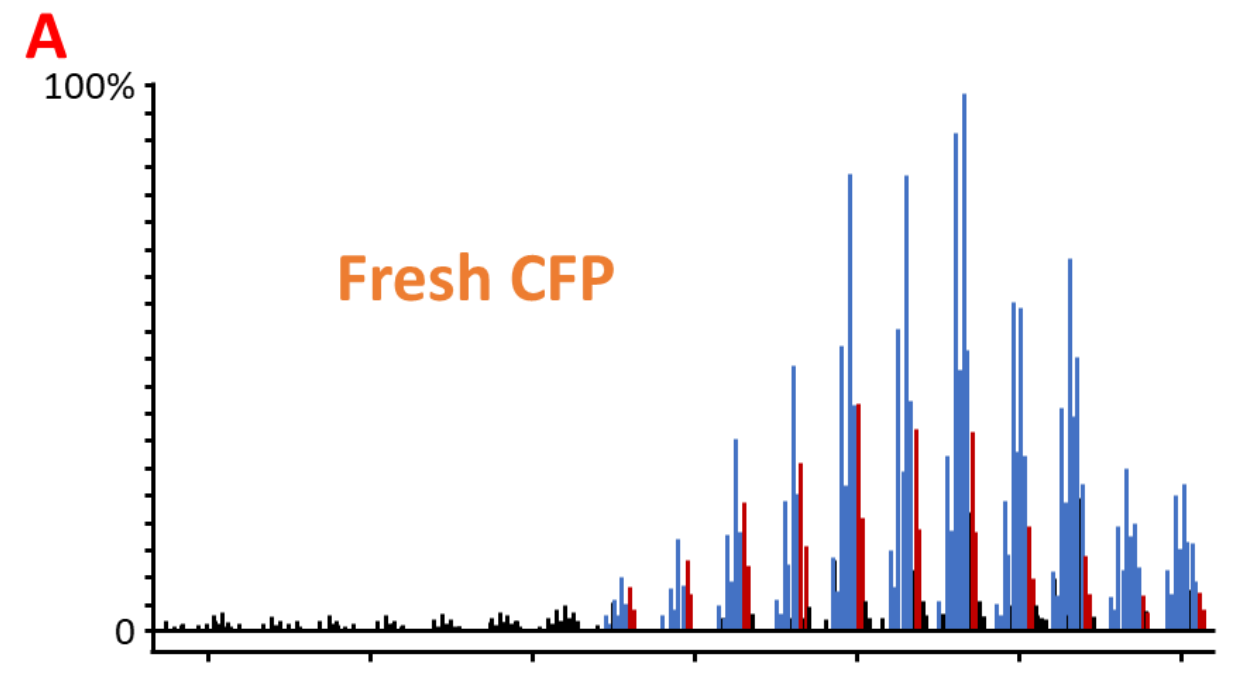

B

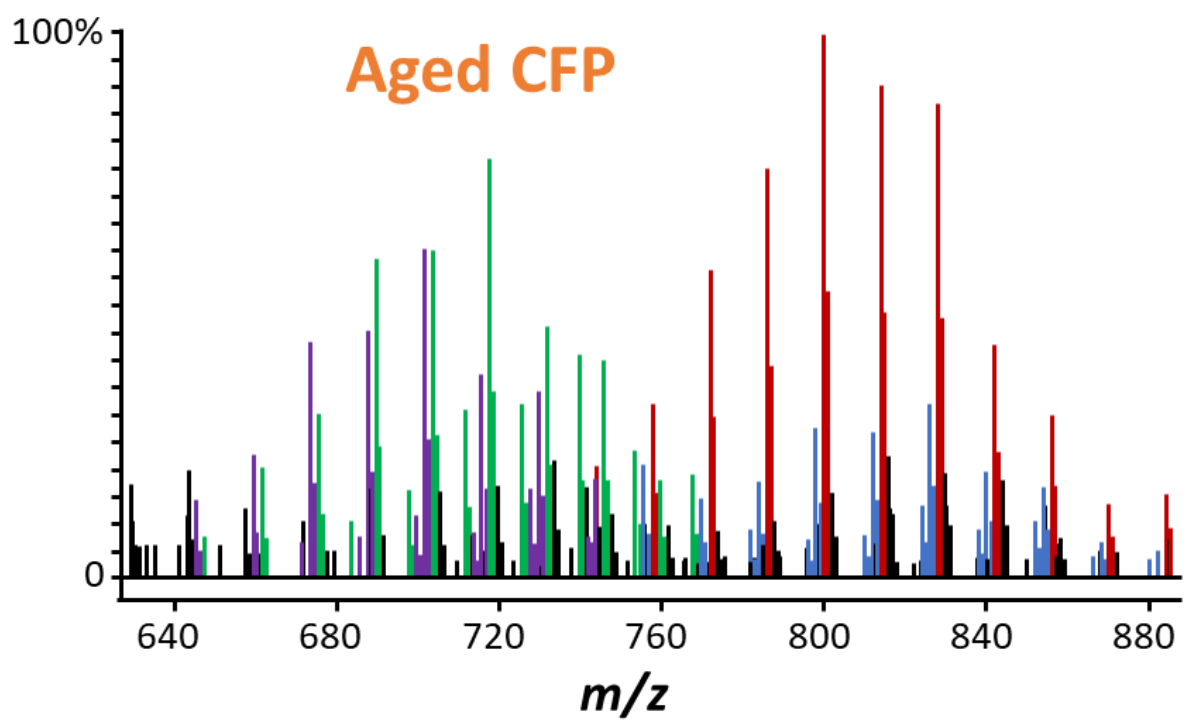

Unsaturated TGs, 6 Oxygens $\square$ Saturated TGs, 6 Oxygens

7 Oxygen Series (Aldehyde) $\square 8$ Oxygen Series (Criegee lons)

Figure S-4. MALDI mass spectra of the TG region from fresh (A) and seven-day aged (B) carbon powder (CFP) developed fingerprints. Note that the CFP was utilized as an existing matrix and no sodium gold was added prior to analysis. 